\title{
Avaliação diagnóstica: uma ferramenta para avaliar a evolução do desempenho dos alunos do Curso de Odontologia do Centro Universitário Newton Paiva
}

\author{
Geraldo Magela Pereira*, Diele Carine Barreto Arantes**, José Flávio Batista Gabrich \\ Giovannini***, Júnia Noronha Carvalhais Amorim*, Santuza Maria de Souza Mendonça*** \\ * Professor Titular do Curso de Odontologia do Centro Universitário \\ Newton Paiva \\ ** Coordenadora do Curso de Odontologia do Centro Universitário \\ Newton Paiva \\ *** Professor Adjunto do Curso de Odontologia do Centro \\ Universitário Newton Paiva
}

\section{RESUMO}

Para a melhoria da qualidade da educação superior e a orientação da expansão do número de Instituições de Ensino Superior (IESs), criou-se, em 14 de abril de 2004, o SINAES - Sistema Nacional de Avaliação da Educação Superior, que representou um avanço significativo na avaliação dos cursos e das IESs, na medida em que permitiu estabelecer padrões de qualidade para a educação superior. $\mathrm{O}$ aprimoramento do processo avaliativo apresenta forte tendência para a busca de consonância entre o olhar do avaliador e os objetivos propostos pelo Projeto Pedagógico do curso a ser avaliado. Esse aprimoramento motiva a criação de ferramentas internas que contemplem as particularidades de cada curso e visem a excelência no processo formativo do discente. Baseado nessas necessidades, a Comissão de Avaliação do Curso de Odontologia do Centro Universitário Newton Paiva elaborou um instrumento para avaliação da evolução do desempenho dos discentes. Essa ferramenta, composta por questões de memorização, análise de dados e elaboração de raciocínios, procurou abranger os diferentes momentos do curso, contemplando conteúdos de conhecimentos básicos, técnicos e articulados. Nesse artigo são apresentadas análises detalhadas dos resultados da avaliação diagnóstica. Constatou-se que a avaliação proporciona contínuo aperfeiçoamento e produz conhecimento que alicerça as tomadas de decisões pelo Núcleo Docente Estruturante. Dentro desse contexto, a Ferramenta Diagnóstica do curso de Odontologia se torna fundamental para o alcance da excelência no processo formativo do discente.

\section{DESCRITORES}

Educação em Odontologia. Ensino. Avaliação Educacional.

$\Delta$ partir da década de 1990 ocorreu um movimento de expansão da educação superior evidenciado pelo aumento significativo de Instituições de Ensino Superior (IESs), o que levou à necessidade de regulamentação e orientação dos cursos superiores no Brasil. ${ }^{1}$ Com o objetivo de fundamentar o planejamento dos projetos pedagógicos destes cursos e superar as concepções tradicionais, a rigidez e o conteudismo dos currículos mínimos, entraram em vigência, em 2002, as diretrizes curriculares nacionais. ${ }^{2}$ Para a melhoria da qualidade da educação superior e a orientação do aumento do número de IESs, criou-se, em 14 de abril de 2004, o SINAES Sistema Nacional de Avaliação da Educação Superior - formado por três componentes principais:

- a avaliação das instituições,

- a avaliação dos cursos e, por fim,

- a avaliação do desempenho dos estudantes.

O SINAES representou uma das ações mais rele- 
Avaliação diagnóstica: uma ferramenta para avaliar a evolução do desempenho dos alunos do Curso de Odontologia do Centro Universitário Newton Paiva • Pereira GM, Arantes DCB, Giovannini JFBG, Amorim JNC, Mendonça SMS

vantes na avaliação dos cursos e das IESs, na medida em que permitiu estabelecer padrões de qualidade para a educação superior e demonstrar a qualidade do processo educativo desenvolvido pelas universidades, sendo denominado por diversos autores como sistema de regulação de educação superior. ${ }^{3} \mathrm{O}$ aprimoramento do processo avaliativo, principalmente baseado nas novas orientações do Ministério da Educação ${ }^{4}$ no que compete à avaliação in loco, apresenta forte tendência para a busca de consonância entre o olhar do avaliador e os objetivos propostos pelo Projeto Pedagógico do curso a ser avaliado.O aprimoramento dos processos avaliativos motiva a criação de ferramentas internas que contemplem as particularidades de cada curso e que visem o alcance da excelência no processo formativo do discente. ${ }^{5}$ Nesse estudo, o enfoque da avaliação será especificamente na aprendizagem, pois é justamente nessa área que são produzidos os efeitos mais marcantes dentro e fora do contexto das instituições educacionais, com repercussões de caráter ético, político e pedagógico. ${ }^{6}$ Baseado nessas necessidades, esse trabalho se propõe a descrever o processo de construção e revelar resultados alcançados com a primeira aplicação de uma ferramenta de avaliação da evolução do desempenho dos discentes do Curso de Odontologia do Centro Universitário Newton Paiva, de acordo com o seu Projeto Pedagógico.

\section{METODOLOGIA}

Em concordância com projeto pedagógico do Curso de Odontologia do Centro Universitário Newton Paiva, a Comissão de Avaliação, constituinte do Núcleo Docente Estruturante desse curso, elaborou um instrumento para avaliação da evolução do desempenho de seus discentes. Essa ferramenta procurou abranger os diferentes momentos do curso, contemplando conteúdos de conhecimentos básicos, técnicos e articulados, através de questões que proporcionassem memorização, análise de dados e elaboração de raciocínios. Tomando-se com base as orientações de Moretto (2005), ${ }^{7}$ foram elaboradas 11 questões objetivas que abordavam os diferentes conteúdos do curso, e também 2 questões discursivas articuladas. Essas questões foram subdivididas em tópicos com conteúdos ministrados em diferentes períodos do curso. Durante o processo de elaboração da ferramenta, os docentes foram mobilizados de forma a garantir a validação das questões, a articulação dos diferentes conteúdos e dos níveis de complexidade de acordo com a dinâmica curricular. A ferramenta elaborada foi aplicada a 427 acadêmicos do curso, independente do período matriculado. As questões foram elaboradas de forma a avaliar a evolução do desempenho dos discentes ao longo do curso, possibilitando identificar as fragilidades e potencialidades dos conteúdos trabalhados. Cada questão foi classificada de acordo com o tema, a área e os períodos cujos conteúdos foram abordados.

Após a aplicação e a correção desta avaliação diagnóstica, cada questão foi analisada quantitativamente, definindo-se o porcentual de acerto em cada período. Posteriormente, comparou-se o resultado de cada questão entre os diferentes períodos. Considerou-se, para as questões objetivas, o número de alunos que respondeu cada alternativa, sendo destacado o número de acertos. Para as questões discursivas, as respostas foram analisadas como:

- correta,

- parcialmente correta,

- errada e/ou em branco.

\section{RESULTADOS E DISCUSSÃO}

Para a melhor compreensão dos resultados, faz-se necessária uma descrição objetiva da estrutura curricular do Curso de Odontologia do Centro Universitário Newton Paiva. Nessa estrutura, as disciplinas básicas são ofertadas nos três primeiros períodos, seguidas pelos conteúdos específicos abordados de forma articulada nas atividades teóricas e nas práticas clínicas dentro das disciplinas de Ciências Odontológicas Articuladas (COAs), que vão do quarto ao nono período.

As COAs ofertam em sua prática clínica o atendimento integral do paciente, com a execução de procedimentos em uma sequência progressiva de complexidade.

A Tabela 1 apresenta os resultados quantitativos de acertos em cada período por grupos de questões objetivas, sendo o Grupo 1 composto por questões de conteúdo básico e o Grupo 2 envolvendo questões técnico-articuladas. Pode-se observar nessa tabela, que os períodos mais avançados $\left(6^{\circ}\right.$ ao $\left.9^{\circ}\right)$ obtiveram o maior percentil de acertos tanto nas questões de conteúdo básico, quanto nas questões de conteúdo técnico-articulado. Esses resultados demonstram que o ensino agregou conhecimentos aos alunos. Para verificar como o conhecimento foi adquirido no decorrer do curso construiu-se o Gráfico 1, onde percebe-se que os alunos se apropriaram de forma progressiva e crescente dos conteúdos básicos e técnico-articulados. Destaca-se que os primeiros períodos $\left(1^{\circ}\right.$ ao $\left.3^{\circ}\right)$ apresentaram melhores resultados em relação à área básica, o que está coerente com o conteúdo trabalhado até esse 
Avaliação diagnóstica: uma ferramenta para avaliar a evolução do desempenho dos alunos do Curso de Odontologia do Centro Universitário Newton Paiva • Pereira GM, Arantes DCB, Giovannini JFBG, Amorim JNC, Mendonça SMS

Tabela 1 - Porcentual de acertos das questões objetivas.

\begin{tabular}{|c|c|c|c|c|c|c|c|}
\hline Grupo & $\begin{array}{l}\text { Conteúdos } \\
\text { abordados }\end{array}$ & Período & $\%$ Acerto & Período & $\%$ Acerto & Período & $\%$ Acerto \\
\hline \multirow{3}{*}{1} & \multirow{3}{*}{ Básicos } & 10 & 34,64 & 40 & 40,74 & 70 & 44,83 \\
\hline & & $2 \mathrm{O}$ & 40,68 & 50 & 45,91 & $8^{\circ}$ & 45,55 \\
\hline & & 30 & 39,98 & 60 & 46,18 & 90 & 52,97 \\
\hline \multirow{3}{*}{2} & \multirow{3}{*}{ Técnico-articulados } & 1 은 & 25,45 & 40 & 50,53 & 70 & 67,16 \\
\hline & & $2^{\circ}$ & 28,21 & 5 은 & 41,16 & 80 & 60,59 \\
\hline & & 30 & 31,85 & 60 & 61,14 & 90 & 58,19 \\
\hline \multirow{3}{*}{3} & \multirow{3}{*}{$\begin{array}{l}\text { Todos (básicos }+ \\
\text { técnico-articulados) }\end{array}$} & $1 \stackrel{0}{1}$ & 30,04 & $4{ }^{\circ}$ & 45,63 & $7^{0}$ & 55,99 \\
\hline & & 20 & 34,44 & 5 은 & 43,53 & 80 & 53,07 \\
\hline & & 30 & 35,91 & 60 & 53,66 & 90 & 55,58 \\
\hline
\end{tabular}

(\%)

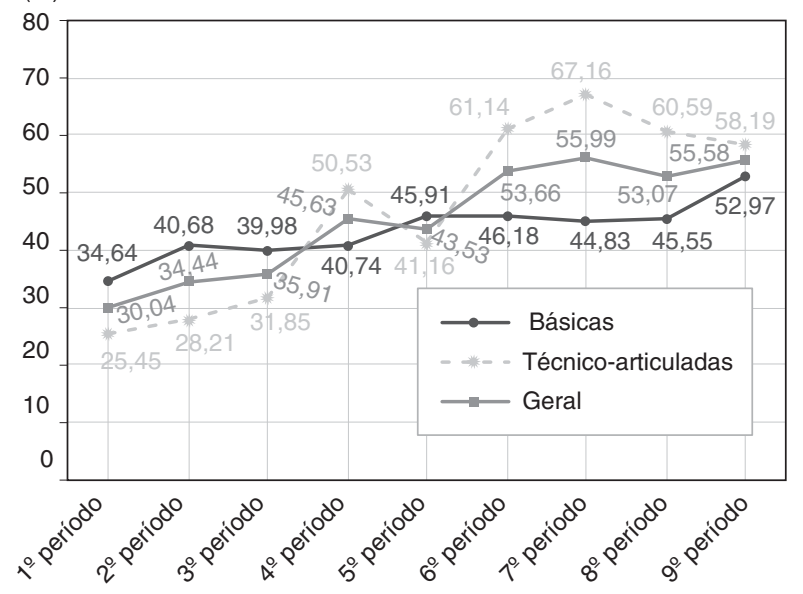

Gráfico 1 - Desempenho dos alunos nas questões fechadas de conhecimento básico, técnico e articulado.

momento do curso. Outra observação interessante é que o desempenho dos alunos em relação ao conteúdo básico seguiu uma progressão contínua e tênue demonstrando assimilação dos conteúdos, o que pode estar relacionado com a aplicação destes conhecimentos nas diferentes clínicas articuladas.

Em relação ao conteúdo articulado, houve oscilação ao longo do curso. Pode-se observar uma progressão acentuada no número de acertos do $3^{\circ}$ para o $4^{\circ}$ período, coincidindo com o início das COAs. Em seguida nota-se uma queda acentuada no $5^{\circ}$ período, posterior progressão até o $7^{\circ}$. No $8^{\circ}$ e $9^{\circ}$ períodos, o gráfico apresentou um declínio tênue. Tais oscilações podem ser explicadas pelo envolvimento das turmas na resolução das questões da ferramenta, pelas diferenças inerentes a cada turma, além das diversas experiências vivenciadas nas clínicas integradas por cada uma delas. Sugere-se também que o momento em que o aluno vivencia a aplicação clínica dos conhecimentos teóricos adquiridos possa in- fluenciar esta avaliação. Isso ficou evidente pelo declínio tênue observado no $8^{\circ}$ e $9^{\circ}$ períodos, quando os conteúdos avaliados nas questões não são abordados de forma explícita em aulas teóricas e práticas.

Este achado indica a necessidade de aplicação periódica da ferramenta diagnóstica com o intuito de obter um histórico de avaliação com dados mais específicos do desempenho das diferentes turmas, nos diferentes momentos do curso. Além disso, a continuidade na aplicação da ferramenta diagnóstica possibilitará o monitoramento do aprendizado dos alunos em um curso onde todas as atividades clínicas são integradas. A proposta de clínicas integradas está contemplada nas diretrizes curriculares nacionais (Perri de Carvalho, 2008) e flexibiliza a formação dos alunos, pois permite experiências diferentes em uma mesma atividade clínica. Porém, o resultado deve ser monitorado para garantir o aprendizado de habilidades básicas e fundamentais na formação de um cirurgião-dentista generalista.

A Tabela 2 apresenta os resultados referentes à primeira questão discursiva, que contemplou o conteúdo de Saúde Coletiva. Pode-se observar que houve aumento significativo de acertos no $2^{\circ}$ período, coincidindo com a disciplina de Estágio II, que aborda especificamente o conteúdo avaliado. A partir daí, observa-se estabilidade do número de acertos até o $7^{\circ}$ período. Como no $8^{\circ}$ e no $9^{\circ}$ períodos, volta a ser abordado o conteúdo de saúde coletiva, pode-se notar um aumento no número de acertos.

Quanto à segunda questão, que aborda conteúdos clínico-articulados, até o $3^{\circ}$ período não houve acertos, o que está coerente com os conteúdos ministrados até o momento. A partir daí, houve um aumento progressivo no número de acertos. Entretanto, a questão que aborda o conteúdo de Semiologia/Farmacologia apre- 
Avaliação diagnóstica: uma ferramenta para avaliar a evolução do desempenho dos alunos do Curso de Odontologia do Centro Universitário Newton Paiva • Pereira GM, Arantes DCB, Giovannini JFBG, Amorim JNC, Mendonça SMS

Tabela 2 - Resultado das respostas dos alunos às questões abertas que abordaram o conteúdo de Saúde Coletiva.

\begin{tabular}{|c|c|c|c|c|c|c|c|}
\hline Questão & $\begin{array}{l}\text { Conteúdo } \\
\text { abordado }\end{array}$ & Período & $\%$ Acerto & Período & $\%$ Acerto & Período & $\%$ Acerto \\
\hline \multirow{3}{*}{$1 \mathrm{~A}$} & \multirow{3}{*}{$\begin{array}{l}\text { Saúde } \\
\text { Coletiva }\end{array}$} & 10 & 1,29 & $4^{\circ}$ & 30,95 & 70 & 9,75 \\
\hline & & $2^{\circ}$ & 38,46 & $5^{\circ}$ & 25,00 & 80 & 37,93 \\
\hline & & 30 & 17,56 & 60 & 28,57 & 90 & 42,85 \\
\hline \multirow{3}{*}{ 1B } & \multirow{3}{*}{$\begin{array}{l}\text { Saúde } \\
\text { Coletiva }\end{array}$} & 10 & 0,00 & 4 은 & 2,38 & 70 & 17,07 \\
\hline & & $2^{\circ}$ & 9,61 & 50 & 10,41 & 80 & 20,68 \\
\hline & & 3 음 & 11,26 & 60 & 2,85 & $9 \underline{-}$ & 14,28 \\
\hline \multirow{3}{*}{$1 \mathrm{C}$} & \multirow{3}{*}{$\begin{array}{c}\text { Saúde } \\
\text { Coletiva }\end{array}$} & 10 & 1,29 & $4^{\circ}$ & 26,19 & 70 & 31,70 \\
\hline & & 20 & 26,92 & $5^{\circ}$ & 33,33 & $8^{\circ}$ & 34,48 \\
\hline & & 3 은 & 27,02 & 60 & 40,00 & 9 응 & 46,42 \\
\hline
\end{tabular}

Tabela 3 - Resultado das respostas dos alunos às questões abertas, divididas de acordo com o conteúdo abordado.

\begin{tabular}{|c|c|c|c|c|c|c|c|}
\hline Questão & $\begin{array}{l}\text { Conteúdo } \\
\text { abordado }\end{array}$ & Período & $\%$ Acerto & Período & $\%$ Acerto & Período & $\%$ Acerto \\
\hline \multirow{3}{*}{$2 A$} & \multirow{3}{*}{ Endodontia } & 10 & 0,00 & $4^{\circ}$ & 16,66 & 70 & 92,68 \\
\hline & & $2^{\circ}$ & 0,00 & 50 & 29,16 & $8^{\circ}$ & 75,86 \\
\hline & & 3으 & 0,00 & 60 & 88,57 & 9o & 92,85 \\
\hline \multirow{3}{*}{$2 B$} & \multirow{3}{*}{ Endodontia } & 10 & 0,00 & 40 & 33,33 & $7^{\circ}$ & 53,65 \\
\hline & & $2^{\circ}$ & 0,00 & 5 웅 & 31,25 & $8^{\circ}$ & 75,86 \\
\hline & & 3 은 & 4,05 & 6음 & 45,71 & 9 응 & 96,42 \\
\hline \multirow{3}{*}{$2 \mathrm{C}$} & \multirow{3}{*}{ Prótese } & 10 & 0,00 & 40 & 0,00 & $7^{\circ}$ & 34,14 \\
\hline & & $2^{\circ}$ & 0,00 & $5^{\circ}$ & 0,00 & 8 은 & 20,68 \\
\hline & & 30 & 0,00 & 60 & 5,71 & 90 & 50,00 \\
\hline \multirow{3}{*}{$2 D$} & \multirow{3}{*}{ Periodontia } & 1 은 & 0,00 & 40 & 0,00 & $7^{\circ}$ & 43,90 \\
\hline & & $2^{\circ}$ & 0,00 & 5 웅 & 10,41 & $8^{\circ}$ & 31,03 \\
\hline & & 3으 & 0,00 & 60 & 17,14 & 9응 & 75,00 \\
\hline \multirow{3}{*}{$2 \mathrm{E}$} & \multirow{3}{*}{$\begin{array}{l}\text { Periodontia/ } \\
\text { Prótese }\end{array}$} & 10 & 0,00 & 40 & 4,76 & 70 & 90,24 \\
\hline & & $2^{\circ}$ & 1,92 & 50 & 27,08 & $8^{\circ}$ & 86,20 \\
\hline & & 3 웅 & 0,00 & 60 & 91,42 & 9 은 & 82,14 \\
\hline \multirow{3}{*}{$2 \mathrm{~F}$} & \multirow{3}{*}{$\begin{array}{c}\text { Semiologia/ } \\
\text { Farmacologia }\end{array}$} & 10 & 0,00 & $4^{\circ}$ & 9,52 & $7^{\circ}$ & 2,43 \\
\hline & & 20 & 0,00 & 50 & 10,41 & $8^{\circ}$ & 0,00 \\
\hline & & 3으 & 0,00 & 60 & 11,42 & 9응 & 3,57 \\
\hline
\end{tabular}

sentou baixo índice de acerto em todos os períodos, merecendo destaque como um provável ponto de fragilidade na dinâmica curricular. Este dado novamente indica a necessidade de aplicação periódica da Ferramenta Diagnóstica com o intuito de obter um histórico de avaliações que possam validar tal questionamento.

No Gráfico 2 estão apresentados em conjunto os resultados obtidos nas duas questões discursivas aplicadas.

Após a análise de toda a Ferramenta Diagnóstica pela Comissão de Avaliação, os dados foram apresentados ao corpo docente para sua análise e discussão com a finalidade de identificar as potencialidades e fragilidades do processo ensino-aprendizagem, possibilitando as discussões necessárias a fim de garantir seu aprimoramento constante.

\section{CONSIDERAÇÕES FINAIS}

A avaliação ocupa, sem dúvida, espaço relevante no conjunto das práticas pedagógicas aplicadas ao processo de ensino e aprendizagem. Não há como fugir da necessidade de avaliação quantitativa de conhecimentos. Contudo, os mecanismos avaliativos devem também verificar a qualidade do processo de ensino e aprendizagem, mostrando as potencialida- 
Avaliação diagnóstica: uma ferramenta para avaliar a evolução do desempenho dos alunos do Curso de Odontologia do Centro Universitário Newton Paiva • Pereira GM, Arantes DCB, Giovannini JFBG, Amorim JNC, Mendonça SMS

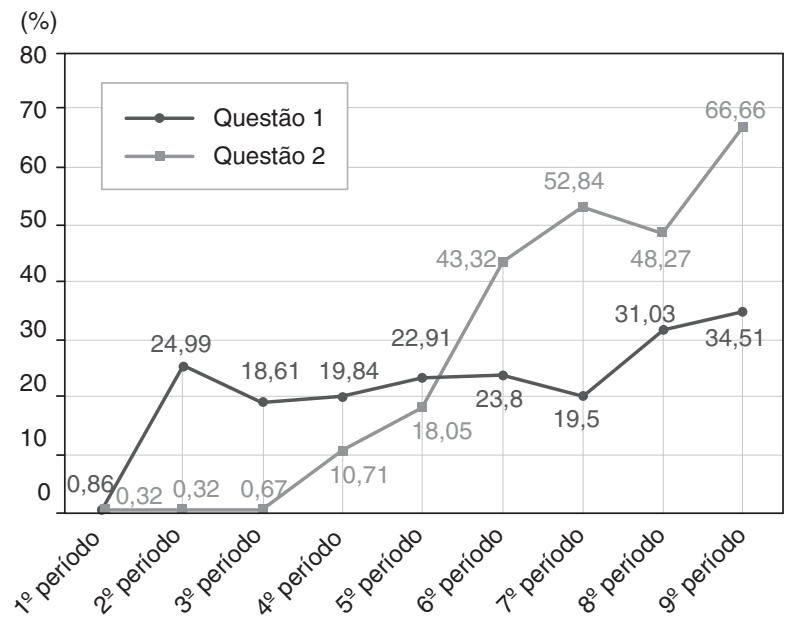

Gráfico 2 - desempenho dos alunos nas questões abertas.

des e fragilidades, e reorientando o trabalho de todo o processo de ensino baseado no projeto pedagógico de cada IES. Portanto, a avaliação orientada para a melhoria da qualidade proporciona contínuo aperfeiçoamento e produz conhecimento que alicerça as tomadas de decisões pelo Núcleo Docente Estruturante. Baseado nessas necessidades, a aplicação periódica da Ferramenta Diagnóstica do curso de Odontologia se torna fundamental para o alcance da excelência no processo formativo do discente.

\section{ABSTRACT}

Diagnostic evaluation: A tool to evaluate the progression of student performance in the Dentistry Course of The Newton Paiva University Center

SINAES (National Higher Education Evaluation System) was established on April 14, 2004, to improve the quality of higher education and serve as a guide for expanding the number of Higher Education Institutions (HEIs). SINAES represents a significant advance in HEI course evaluation, insofar as it has made it possible to establish quality standards for higher education. The improvement in the assessment process has shown a strong trend toward matching the views of the assessor with the objectives proposed by the educational project of the course to be assessed. This improvement drives the effort to create internal tools that address the peculiarities of each course and that aim at achieving excellence in the students' educational process. Based on these needs, the Evaluation Committee of the Newton Paiva University Cen- ter Dentistry Course designed a tool to evaluate the progression of student performance. This tool consists of questions related to memorization, data analysis and development of reasoning processes. It seeks to cover the different moments of the course, taking into account contents related to basic, technical and interrelated knowledge. This article presents in-depth analyses of the results of the diagnostic evaluation. It was found that an evaluation process promotes continuous improvement and produces knowledge that shores up decision-making processes by the Structuring Faculty Core. As such, the Diagnostic Tool of the dentistry course plays a fundamental role in steering the student to achieve educational excellence.

\section{DESCRIPTORS}

Education, Dental. Teaching. Educational Mesurement. :

\section{REFERÊNCIAS}

1. Morita MC; Haddad AE; Araújo ME. Perfil atual e tendências do cirurgião-dentista brasileiro. Maringá: Dental Press International; 2010.

2. Perri de Carvalho AC. Reforma curricular da Odontologia. In: Botazzo, C; Oliveira, MA. Atenção básica no sistema único de saúde: abordagem interdisciplinar para os serviços de saúde bucal. Cap. 16. São Paulo: Páginas \& Letras Editora e Gráfica, 2008

3. Alonso MS; Antoniazzi JH. Livro do projeto Latino-Americano de convergência em educação odontológica (PLACEO). São Paulo: Artes Médicas; 2010.

4. Documento orientador das Comissões de Avaliação IN LOCO: Ministério da Educação, INEP Março, 2012.

5. Albino JEN; Young SK; Neumann LM; Kramer GA; Andrieu SC; Henson L; Horn B; Hendricson WD. Assessing dental students' competence: best practice recommendations in the performance assessment literature and investigation of current practices in predoctoral dental education. Journal of Dental Education 2008;72(12):1405-1435.

6. Masetto MT; Prado AS. Processo de avaliação da aprendizagem em curso de odontologia. Revista da ABENO 2004;4(1):48-56.

7. Moretto V. Prova - um momento privilegiado de estudo - não um acerto de contas. 6a ed. Rio de Janeiro: DP\&A, 2005.

Recebido em 08/10/2012

Aceito em 10/12/2012 